\title{
AFRL-OSR-VA-TR-2013-0144
}

Geometry, Representation Theory, and the Langlands Program

Kari Vilonen

Northwestern University

April 2013

Final Report

DISTRIBUTION A: Approved for public release.

\author{
AIR FORCE RESEARCH LABORATORY \\ AF OFFICE OF SCIENTIFIC RESEARCH (AFOSR) \\ ARLINGTON, VIRGINIA 22203 \\ AIR FORCE MATERIEL COMMAND
}




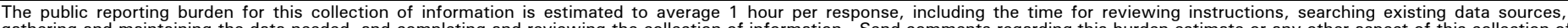

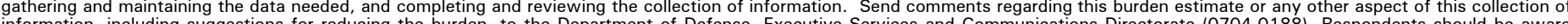

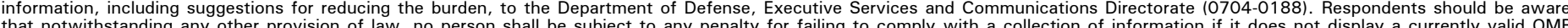

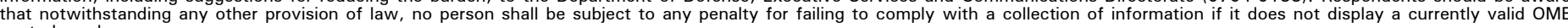
control number.

PLEASE DO NOT RETURN YOUR FORM TO THE ABOVE ORGANIZATION.
1. REPORT DATE $(D D-M M-Y Y Y Y)$ 2. REPORT TYPE $13 / 02 / 2013$
FINAL REPORT
3. DATES COVERED (From - To)
$16 / 6 / 2008-31 / 09 / 2012$

4. TITLE AND SUBTITLE

Geometry, Representation Theory, and the Langlands Program

5a. CONTRACT NUMBER

5b. GRANT NUMBER

FA9550-08-1-0351

5c. PROGRAM ELEMENT NUMBER

6. AUTHOR(S)

5d. PROJECT NUMBER

Kari Vilonen

5e. TASK NUMBER

5f. WORK UNIT NUMBER

7. PERFORMING ORGANIZATION NAME(S) AND ADDRESS(ES)

Northwestern University

Evanston, IL 60208

9. SPONSORING/MONITORING AGENCY NAME(S) AND ADDRESS(ES)

AFOSR

875 North Randolph Street

Suite 325, Rm 3112

Arlington, VA 22203
8. PERFORMING ORGANIZATION REPORT NUMBER

10. SPONSOR/MONITOR'S ACRONYM(S)

11. SPONSOR/MONITOR'S REPORT NUMBER(S)

AFRL-OSR-VA-TR-2013-0144

\section{DISTRIBUTION/AVAILABILITY STATEMENT}

DISTRIBUTION A: APPROVED FOR PUBLIC RELEASE

\section{SUPPLEMENTARY NOTES}

\section{ABSTRACT}

Schmid and Vilonen have mostly carried out the program of determining the Unitary dual of reductive Lie groups using Hodge theory. Kashiwara and Vilonen solved a long standing conjecture on the microlocal structure of holonomic systems of differential equations, the codimension-three conjecture. Bezrukavnikov has carried out a far reaching generalization of Kazhdan-Lustzig combinatorics to a categorical level. Emerton has made fundamental progress in the p-adic Langlands program by proving a strong local-global compatibility.

15. SUBJECT TERMS

\begin{tabular}{|c|c|c|}
\hline \multicolumn{3}{|c|}{ 16. SECURITY CLASSIFICATION OF: } \\
\hline a. REPORT & b. ABSTRACT & c. THIS PAGE \\
U & U & U \\
\hline
\end{tabular}

\section{LIMITATION OF} ABSTRACT

SAR
18. NUMBER

OF

PAGES 19a. NAME OF RESPONSIBLE PERSON

Kari Vilonen

19b. TELEPHONE NUMBER (Include area code) 


\title{
FINAL REPORT GEOMETRY, REPRESENTATION THEORY, AND THE LANGLANDS PROGRAM
}

\author{
KARI VILONEN
}

\section{Summary}

The work under this contract was performed by many people and under some subcontracts. The report is divided into separate sections according to the subcontracts.

\section{WORK OF KaRI VILONEN}

The main work of the PI during the grant period focused on two longstanding fundamental problems in two separate areas of mathematics. The first one is the question of the unitary dual for reductive Lie groups. This question has remained open for well over 50 years despite the efforts of many people. The PI and Wilfried Schmid (Harvard) have developed a completely novel approach to this problem. We approach this problem from a geometric point of view making use of the theory of mixed Hodge Modules of Saito. Our approach and conjectures are outlined in publication 1.

The second fundamental problem the PI attacked with M. Kashiwara (RIMS, Kyoto). We solved the main conjecture on the micro local structure of holonomic systems of differential equations. Our work leads to an interesting extension of the theory of several complex variables. Conjecturally one can extend the basic Oka-Cartan theory to an arbitrary Noetherian DNF base. Our results have been written up.

There is also a joint project with Bezrukavnikov where we prove a categorical Langlands duality for real groups. The paper is under preparation. The PI also is working on a project with Williamson (MPI, Bonn) on the Lustzig conjecture on modular representations.

\section{The list of publications.}

1. Kashiwara and Vilonen, On the codimension-three conjecture, Proc. Jpn. Acad., Ser. A 86 (2010), 154158.

2. Schmid and Vilonen, Hodge theory and unitary representations of reductive Lie groups, Frontiers of Mathematical Sciences, International Press 2011, 397-420. 
3. Kashiwara and Vilonen, Microdifferential systems and the codimensionthree conjecture, preprint, arXiv:1209.5124, in the process of being revised for the Annals.

4. K.Vilonen and G.Williamson, Characteristic cycles and decomposition numbers, preprint, arXiv:1208.1198

5. Bezrukavnikov and Vilonen, Langlands duality for real groups, manuscript almost complete.

6. Taskinen and Vilonen, An extension of Cartan-Oka theory, in preparation.

7. Schmid and Vilonen, Hodge theory and real groups, a series of papers are in preparation.

\section{WORK OF DEEM}

Deem and his collaborators derive a 'thermodynamic' formulation of evolutionary dynamics. Euler's theorem for homogeneous functions allowed them to achieve the needed Legendre-like transformation.

\section{The list of publications.}

(1) Muoz, Enrique; Park, Jeong-Man; Deem, Michael W., Solution of the Crow-Kimura and eigen models for alphabets of arbitrary size by Schwinger spin coherent states. J. Stat. Phys. 135 (2009), no. 3, 429-465.

\section{IAS SubCONTRACT}

This grant supported the following people, who did research at the Institute for Advanced Study: CHAUDOUARD, FARGUES, GORESKY, NAIR, and NGO.

The mathematical context of the work was this: The Langlands program has classically been rather un-functorial (despite the name of Langlands functoriality) and un-geometric. It has has seen two notable advances recently, both tying it to geometry:

1. Geometric Langlands (Beilinson, Drinfeld, Lauman, Vilonen, and others). The Langlands program for function fields has been reformulated in a way that is both geometric and functorial.

2. The Fundamental Lemma (Proved by Ngo, following initial ideas of GoreskyKottwitz-MacPherson and Laumon). Lack of proof of this had been the main obstacle to completing many instances of the Langlands program. The proof of the Fundamental Lemma is also geometric. It involves geometry related to, but not identical to, that of geometric Langlands.

A great opportunity now exists to unify these two threads of progress. Whatever synthesis comes out of them will surely be very far reaching. That is the greater vision towards which this project was leading.

A group of leaders in the field assembled at the Institute for Advanced Study. Key members of this group were supported by this DARPA grant. Others already had support from other means. (For example, Morel had a Clay fellowship, Yun 
was a graduate student of Princeton University, and Langlands is an IAS Faculty member).

A seminar was organized in the Institute in 2008-9. Chaudouard explained his proof with Laumon of the weighted fundamental lemma which is also necessary to Arthur's stabilization of the trace formula, Morel and Shin gave new and almost optimal construction of Galois representations attached to auto-dual automorphic representations in the cohomology of Shimura varieties, Lan talked about his construction of compactification of Shimura varities over the integers, Getz explained new ideas about periods, distinguished representations and relative trace formula, Yun about the moduli space of parabolic Higgs bundles and his construction of global Springer representations and Fargues his new ideas on a reduction theory for the moduli space of $p$-divisible groups.

\section{BEZRUKAVNIKOV}

During the grant period Bezrukavnikov has completed two major projects related to the grant subject and a number of smaller ones. He has also initiated several new directions, partly based on the previous results.

In one completed project (partly joint with I. Mirkovic and others) ideas of local geometric Langlands duality are applied to investigate categorification of standard modules for the affine Hecke algebra. This is used to derive a far generalization of Kazhdan-Lusztig Conjectures describing numerical invariants of representations of semi-simple Lie algebras in positive characteristic (the so called "Lusztig's Hope") and oh highest weight modules over affine Lie algebras at the critical level. Ideas of that project play an important role in the new investigation of the relation of geometric Langlands duality to $t$-structures, stabilities and quantum cohomology, partly joint with A. Okounkov (in progress).

In another direction (partly joint with Finkelberg and Ostrik) Bezrukavnikov developed a new approach to classification of character sheaves based on the idea of categorification of the center. In the ongoing work (partly joint with Kazhdan and Varshavsky) these ideas are applied to loop group leading to a new algebro-geometric understanding of the endoscopy phenomenon. This is expected to explain the relation between local geometric Langlands duality, the work of Ngo et al. and endoscopy conjectures in classical Langlands duality theory.

\section{The list of publications by Bezrukavnikov prepared and/or published during the grant period.}

(1) (with D. Kaledin) Fedosov quantization in positive characteristic, J. Amer. Math. Soc. 21 (2008), no. 2, 409-438.

(2) (with S. Arkhipov) Perverse sheaves on affine flags and Langlands dual group, Israel J. Math., 170 (2009), 135-184. 
(3) Perverse sheaves on affine flags and nilpotent cone of the Langlands dual group, Israel J. Math., 170 (2009), 185-206.

(4) (with M. Finkelberg, V. Ostrik) On tensor categories attached to cells in affine Weyl groups, III, Isr. J. Math, 170 (2009), 207-234.

(5) (with M. Finkelberg and V. Ginzburg) Cherednik algebras and Hilbert schemes in characteristic p. With an appendix by Pavel Etingof. Represent. Theory 10 (2006), 254-298

(6) (with M. Finkelberg) Equivariant Satake Category and Kostant-Whittaker reduction, Moscow Math. J. 8 (2008), no. 1, 39-72, 183.

(7) (with P. Etingof) Parabolic induction and restriction functors for rational Cherednik algebras, Selecta Math. (N.S.) 14 (2009), no. 3-4, 397-425.

(8) (with L. Positselski) On semi-infinite cohomology of finite dimensional graded algebras, Compositio Math 146 (2010), no 2, 480-496.

(9) (with D. Arinkin) Perverse coherent sheaves, Moscow Math J. 10 (2010) no 1, 3-29.

(10) (with M. Finkelberg and V. Ostrik) Character D-modules via Drinfeld center of Harsih-Chandra bimodules, Inventiones Math. 188 (2012) no 3, 589-620.

(11) (with I. Mirković, and an Appendix by E. Sommers ) Representations of semisimple Lie algebras in prime characteristic and noncommutative Springer resolution, preprint arXiv:1001.2562, to appear in Annals Math.

(12) (with Z. Yun) On Koszul duality for Kac-Moody groups, Represent. Theory 17 (2013), 1-98.

(13) (with S. Riche) Affine braid group actions on derived categories of Springer resolutions, Annales Scientifiques de l'Ecole Normale Supérieure 45 (2012), 535-599.

(14) (with Q. Lin) Highest weight modules at the critical level and noncommutative Springer resolution, Contemp. Math. 565 (2012) 15-27. 


\section{Emerton Research Accomplishments}

\section{Papers and preprints}

Both the published papers and those unpublished preprints that are fully written are available at www. math.uchicago.edu/ emerton/preprints.html.

[1] F. Calegari, M. Emerton, Mod p cohomology growth in p-adic analytic towers of 3-manifolds, Groups, Geometry, and Dynamics, vol. 5 (2011), pp. 355-366.

[2] F. Calegari, M. Emerton, Completed cohomology - a survey, Non-abelian fundamental groups and Iwasawa theory (J. Coates, M. Kim, F. Pop, M. Saïdi, P. Schneider eds.), London Math. Society Lecture Notes Series, vol. 393 (2012), pp. 239-257.

[3] F. Calegari, M. Emerton, Hecke operators on stable cohomology, preprint, 9 pages.

[4] F. Calegari, M. Emerton, Completed cohomology of arithmetic groups, in preparation.

[5] M. Emerton, Local-global compatibility in the p-adic Langlands programme for $\mathrm{GL}_{2 / \mathbb{Q}}$, preprint, 119 pages.

[6] M. Emerton, p-adic families of modular forms [after Hida, Coleman, and Mazur], Séminaire Bourbaki, 2009/2010, exposé 1013, Astérisque, vol. 339 (2011), 31-61.

[7] M. Emerton, T. Gee, A geometric perspective on the Breuil-Mézard conjecture, to appear in the Journal of the Institute of Mathematics of Jussieu, 34 pages.

[8] M. Emerton, T. Gee, p-adic Hodge-theoretic properties of étale cohomology with mod $p$ coefficients, and the cohomology of Shimura varieties, preprint, 39 pages.

[9] M. Emerton, T. Gee, On the non-vanishing of the universal unitary completions of certain locally algebraic representations of $\mathrm{GL}_{n}$ of a p-adic field, in preparation.

[10] M. Emerton, T. Gee, F. Herzig, Weight cycling and Serre-type conjectures for unitary groups, preprint, 66 pages.

[11] M. Emerton, T. Gee, D. Savitt, Lattices in the cohomology of Shimura curves, in preparation.

[12] M. Emerton, D. Helm, The local Langlands correspondence for $\mathrm{GL}_{n}$ in families, preprint, 61 pages.

[13] M. Emerton, V. Paskunas, On the density of trianguline potentially Barsotti-Tate points in deformation spaces of two-dimensional Galois representations, in preparation.

[14] M. Emerton, R. Pollack, T. Weston, Explicit reciprocity laws and Iwasawa theory for modular forms, in preparation.

\section{Discussion of results}

The paper [2] (joint with Frank Calegari) summarizes our main results and conjectures on the $p$-adically completed cohomology of congruence subgroups of reductive groups over $\mathbb{Q}$. The overall goal of this theory is to present a framework in which to study $p$-adic aspects of the theory of automorphic forms, and its relationship to Galois representations, which takes into account torsion phenomena in cohomology; applies in non-Shimura variety contexts; and allows one to incorporate representation-theoretic methods. Detailed proofs of the results and examples discussed are being written up in the paper [4]. The paper [1] gives applications of our methods to the growth of cohomology in towers of 3-manifolds. 
The most successful application of the ideas of completed cohomology so far has been in the paper [5], in which I prove a strong local-global compatibility statement for the $p$-adically completed cohomology of modular curves, in terms of the $p$-adic local Langlands correspondence. (This work was reported on in the recent Bourbaki seminars of Berger and Breuil.) This result has many cases of the Fontaine-Mazur conjecture for odd two-dimensional Galois representations as a consequence. In studying local-global compatibility in $p$-adically completed cohomology, it is important to consider what happens at all primes, not just the prime $p$, and so as well as the $p$-adic Langlands correspondence, one needs a $p$-adic interpolation of the usual local Langlands correspondence for $\mathrm{GL}_{2}\left(\mathbb{Q}_{\ell}\right)$ for primes $\ell \neq p$. This is provided by the paper [12] (joint with David Helm), which in fact discusses the more general problem of $p$-adically interpolating the local Langlands correspondence for $\mathrm{GL}_{n}(F)$ for any $n$ and any local field $F$ of residue characteristic different from $p$.

One of the difficulties in studying $p$-adically completed cohomology in more general settings is that one has to consider the possible contributions of $p$-power torsion cohomology classes (there are none such for the cohomology of curves, but this won't be the case in higher dimensional contexts). When considering cohomology of congruence subgroups with characteristic zero coefficients, Arthur's conjectures and related ideas suggest that outside the middle dimension (in the Shimura variety case), or the middle range of dimensions (in the non-Shimura variety case), the systems of Hecke eigenvalues that appear are all Eisenstein. Optimistically, one might hope that the same is true for cohomology with mod $p$ coefficients. The papers [3] and [8] both give some evidence for this hope.

In [3] (joint with Frank Calegari), we prove that if $2 d+6 \leq n$, then any Hecke eigenclass in the degree $d$ cohomology of a congruence subgroup with characteristic $p$ coefficients of $\mathrm{GL}_{n}(\mathbb{Q})$ is Eisenstein. (The characteristic zero analogue follows directly from Borel's proof of stability of cohomology with characteristic zero coefficients, via an automorphic argument which is not applicable in characteristic $p$.) The argument combines results on stability of cohomology for congruence subgroups with a representation-theoretic argument exploiting lower bounds on the Gelfand-Kirillov dimensions of representations of $\mathrm{GL}_{n}\left(\mathbb{Q}_{\ell}\right)$ in characteristic $p$.

In [8] we consider congruence subgroups of unitary groups over $\mathbb{Q}$ of type $U(n-1,1)$ at infinity. We prove that if $\mathfrak{m}$ is a maximal ideal in the Hecke algebra acting on the mod $p$ cohomology of such a congruence subgroup, to which is associated a mod $p n$-dimensional Galois representation with sufficiently big image and which satisfies certain technical conditions locally at $p$, then the system of Hecke eigenvalues attached to $\mathfrak{m}$ cannot occur in degrees $i<n / 2$. These results are strongest when $n=2$, since our results then imply that the system of eigenvalues attached to $\mathfrak{m}$ can appear only in the middle degree (i.e. degree $i=2$ ). The most novel aspect of our result is that applies to congruence subgroups with arbitrary powers of $p$ in the level, where traditional methods of integral $p$-adic Hodge theory don't apply. We introduce new methods in integral $p$-adic Hodge theory inspired by ideas of Beilinson and Nori, relying on careful applications of the weak Lefschetz theorem.

The papers [7], [9], and [11] are closely related to the ideas presented in Section 4 of the present proposal, and I will discuss them in more detail in that section.

In the paper [10] (joint with Toby Gee and Florian Herzig), we verify the weight part of Serre's conjecture for certain 3-dimensional mod $p$ global Galois representations which are modular in the sense that they correspond to systems of Hecke eigenvalues arising from mod $p$ algebraic automorphic forms on a unitary group with is of type $U(3)$ at infinity, using a combination of arguments in integral $p$-adic Hodge theory and a novel representation-theoretic argument we call 
"weight cycling". The vanishing results of [8] allow us to extend this result to unitary groups of type $U(2,1)$ at infinity.

The paper [13] (joint with Vytas Paskunas) addresses the question of the density of certain kinds of points in Galois deformation spaces. It is well-known that if $\bar{\rho}: G_{\mathbb{Q}_{p}} \rightarrow \mathrm{GL}_{2}\left(\overline{\mathbb{F}}_{p}\right)$ is a continuous representation, then the set of potentially Barsotti-Tate representations (i.e. potentially crystalline representations with Hodge-Tate weights 0 and 1) is Zariski dense in the deformation space of $\bar{\rho}$, and similarly that the set of crystabelline representations (i.e. representations that become crystalline over an abelian extension of $\mathbb{Q}_{p}$ ) is Zariski dense. Likewise, given an odd continuous representation $\bar{\rho}: G_{\mathbb{Q}} \rightarrow \mathrm{GL}_{2}\left(\overline{\mathbb{F}}_{p}\right)$ (for which $\bar{\rho}_{\mid G_{\mathbb{Q}\left(\zeta_{p}\right)}}$ is irreducible, so that the Taylor-Wiles method applies), it is known that the points that are potentially Barsotti-Tate locally at $p$ are Zariski dense in the deformation space for $\bar{\rho}$ and also that the set of points that are crystabelline locally at $p$ are Zariski dense. In [13] we prove the stronger result that the points that are simultaneously potentially Barsotti-Tate and crystabelline (locally at $p$, in the global case) are Zariski dense in deformation space. The argument in the local case uses the $p$-adic local Langlands correspondence together with representation-theoretic tools; in the global case, we combine these methods with the local-global compatibility result of [5].

In the paper [14] (joint with Rob Pollack and Tom Weston) we prove a conjecture of Mazur and Tate, to the effect that for a cuspidal Hecke eigenform $f$ with coefficients in $\overline{\mathbb{Q}}_{p}$ (and satisfying certain technical hypotheses), the Fitting ideals of the dual Selmer group of the $p$-adic Galois representation $\rho_{f}$ attached to $f$, over the various $p$-power cyclotomic extensions $\mathbb{Q}\left(\zeta_{p^{b}}\right)$ of $\mathbb{Q}$, contain the Mazur-Tate elements (defined in terms of the algebraic parts of the special values of twists of the $L$-function of $f$ ). This is an analogue and generalization of the divisibility of the analytic $L$-function by the algebraic $L$-function that was proved by Kato in the ordinary case. The argument uses techniques of the $p$-adic Langlands program applied to the Galois representation $\rho_{f}$. 